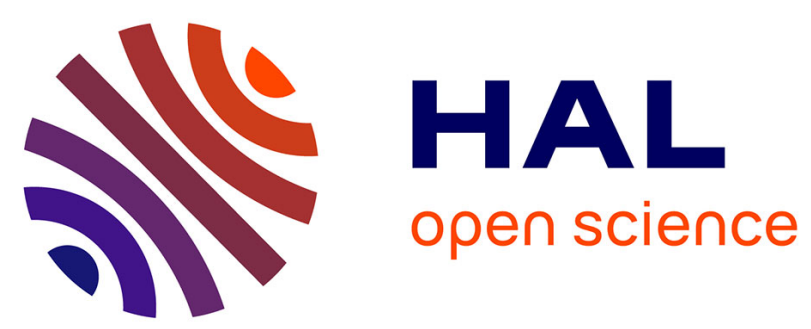

\title{
Selective area molecular beam epitaxy of InSb nanostructures on mismatched substrates
}

L. Desplanque, A. Bucamp, David Troadec, G. Patriarche, X. Wallart

\section{To cite this version:}

L. Desplanque, A. Bucamp, David Troadec, G. Patriarche, X. Wallart. Selective area molecular beam epitaxy of InSb nanostructures on mismatched substrates. Journal of Crystal Growth, 2019, 512, pp.6-10. 10.1016/j.jcrysgro.2019.02.012 . hal-02392434

\section{HAL Id: hal-02392434 \\ https://hal.science/hal-02392434}

Submitted on 21 Oct 2021

HAL is a multi-disciplinary open access archive for the deposit and dissemination of scientific research documents, whether they are published or not. The documents may come from teaching and research institutions in France or abroad, or from public or private research centers.
L'archive ouverte pluridisciplinaire HAL, est destinée au dépôt et à la diffusion de documents scientifiques de niveau recherche, publiés ou non, émanant des établissements d'enseignement et de recherche français ou étrangers, des laboratoires publics ou privés.

\section{다)(1) $(5$}

Distributed under a Creative Commons Attribution - NonCommercial| 4.0 International 


\title{
Selective Area Molecular Beam Epitaxy of InSb nanostructures on mismatched substrates
}

\author{
L.Desplanque $^{* 1}$, A.Bucamp ${ }^{1}$, D.Troadec ${ }^{1}$, G.Patriarache ${ }^{2}$ and X. Wallart $^{1}$ \\ ${ }^{1}$ Univ. Lille, CNRS, Centrale Lille, ISEN, Univ. Valenciennes, UMR 8520 - IEMN, F-59000 \\ Lille, France \\ ${ }^{2}$ C2N, Campus de Marcoussis, CNRS, Université Paris-Saclay, route de Nozay, F-91460 \\ Marcoussis, France \\ *ludovic.desplanque@univ-lille.fr
}

\begin{abstract}
The selective molecular beam epitaxy of $\mathrm{InSb}$ inside nanoscale apertures realized in a $\mathrm{SiO}_{2}$ mask deposited on a highly mismatched substrate is studied. The substrate of interest is GaAs on which a $6.1 \AA$ material (InAs or $\mathrm{AlGaSb}$ ) has been grown accommodating part of the mismatch with InSb. For sub-100 nm wide aperture, several micron long in-plane InSb nanowires can be obtained. Different ways for measuring the electrical properties of these inplane nanostructures are proposed. A $1 \mu \mathrm{m}$ long gate length MOSFET is fabricated on a semiinsulating AlGaSb pseudo-substrate without any transfer on a host substrate.
\end{abstract}

KEYWORDS: A3. Molecular Beam epitaxy; A3. Selective epitaxy; B1.Antimonides; A1.Nanostructures; B3. Field effect transistors; B2. Semiconducting indium compounds

\section{Introduction}

With its remarkable properties (bandgap in the mid-infrared range, high electron mobility, large G-factor and strong spin-orbit coupling), InSb is a key material for electronics, optoelectronics and quantum technologies. However, the elaboration of InSb layers on a semiinsulating substrate requires a metamorphic approach involving threading defect formation 
and a rather low resistivity buffer layer [1]. One other approach consists in growing InSb in the form of vertical nanowires from a (111) substrate and transfer them on a host substrate for device elaboration [2,3]. If high quality material can be obtained, fabricating complex architectures with this process is very tricky [4]. We have demonstrated recently that selective area MBE (SA-MBE) of antimony based materials can be achieved at rather low temperature using an atomic Hydrogen flux during the growth [5]. For instance, GaSb nanostructures on GaAs without threading dislocations have been demonstrated thanks to an accommodation of the mismatch by a regular array of $90^{\circ}$ dislocations at the interface between the nanostructure and the GaAs substrate [6]. These nanostructures can be used as nano-templates to achieve high quality suspended InAs nanowires and fabricate efficient MOSFET [7]. Recent results have shown that the SA-MBE technique is also a useful way to fabricate InAs based ballistic nano-devices for quantum technologies [8,9].

In this work, we show that SA-MBE is also suitable for growing high quality InSb nanostructures on InAs or $\mathrm{AlGaSb}$ pseudo-substrates. The combination of low temperature atomic $\mathrm{H}$ assisted $\mathrm{SA}-\mathrm{MBE}$ and sub-100 nm patterning of the $\mathrm{SiO}_{2}$ mask allows the control of the shape of the nanostructures with the design of the mask apertures. Scanning and transmission electron microscopy as well as MOSFET fabrication are used to investigate the structural and electrical properties of these nanostructures.

\section{Experiments}

Two RIBER MBE systems are used for the growth: a Compact 21 (C21) system equipped with an atomic Hydrogen RF plasma cell and with As and Sb valved crackers for the As and Sb based layers and a RIBER 32P equipped with a phosphine injector for InP growth. The connection under ultra-high vacuum between the two systems allows the growth of P, As and Sb containing layers. Two different pseudo-substrates are first grown on semi-insulating (SI) 
GaAs substrates (figure 1). The InAs pseudo-substrate (Fig. 1a) consists of a $1 \mu \mathrm{m}$ thick InAs layer deposited on the GaAs substrate with an intermediate $30 \mathrm{~nm}$ thick GaSb layer for mismatch accommodation [10]. The AlGaSb pseudo-substrate (Fig. 1b) consists of a $600 \mathrm{~nm}$ thick semi-insulating $\mathrm{Al}_{0.8} \mathrm{Ga}_{0.2} \mathrm{Sb}$ layer grown on the GaAs substrate with 4 monolayers $\mathrm{AlSb}$ in-between [11]. To prevent the oxidation of $\mathrm{AlGaSb}$, a $2.5 \mathrm{~nm}$ thick InP layer is grown on top of the pseudo-substrate. Both pseudo-substrates are then coated with a $30 \mathrm{~nm}$ thick silicon oxide layer by Plasma Enhanced Chemical Vapor Deposition. After patterning the silicon dioxide layer using the process described in reference [12], the samples are re-introduced into the MBE chamber for deoxidization of the semiconductor surface under $\mathrm{As}_{4}$ and atomic hydrogen fluxes for InAs pseudo-substrates and under $\mathrm{Sb}_{2}$ and atomic hydrogen fluxes for $\mathrm{AlGaSb}$ pseudo-substrate. InSb is grown under atomic hydrogen flux at $400^{\circ} \mathrm{C}$ with a rate of 0.2 ML.s ${ }^{-1}$ and a Sb/In flux ratio of 5. Nominal InSb thicknesses of 70 and $100 \mathrm{~nm}$ are deposited on the InAs pseudo-substrates (sample A and B respectively) and a nominal thickness of $70 \mathrm{~nm}$ is deposited on the AlGaSb pseudo-substrate (sample C). After growth, the sample is analyzed using scanning electron microscopy (SEM) and for some nanostructures focused ion beam is used to prepare the samples for scanning transmission electron microscopy (STEM). The STEM studies are performed on a Titan Themis 200 (FEI) microscope equipped with a spherical aberration corrector on the probe and the EDX analysis system super X (0.7 srad solid angle of collection). The accelerative voltage is $200 \mathrm{kV}$. The (half) convergence angle of the probe is $17.6 \mathrm{mrad}$ and the probe current $85 \mathrm{pA}$.

For sample $\mathrm{C}$, the electrical characteristics of some nanostructures are analyzed by the realization of MOSFET using an alumina layer deposited by atomic layer deposition. Details about the fabrication process can be found in reference [13].

\section{Results and discussion}


Figure 2 displays the morphology of sample A for patterns in the $\mathrm{SiO}_{2}$ mask consisting of $20 \mu \mathrm{m}$ long apertures (whose width varies from $1 \mu \mathrm{m}$ down to $50 \mathrm{~nm}$ ) and oriented along [110] (Fig. 2a) and [110] (Fig. 2b) directions, and patterns consisting of an array of holes with a diameter of $100 \mathrm{~nm}$ and a periodicity of $150 \mathrm{~nm}$ (Fig. 2c and 2d). The absence of polycrystalline $\mathrm{InSb}$ deposition on the $\mathrm{SiO}_{2}$ mask reveals a very good growth selectivity. It is achieved thanks to the promotion of In and $\mathrm{Sb}$ re-evaporation from the dielectric surface by atomic hydrogen. The positive impact of an atomic hydrogen flux delivered from a RF plasma source on the deoxidization of InSb surface is known for a long time [14]. The enhanced reevaporation of In and $\mathrm{Sb}$ from the silicon oxide layer may involve a quite similar mechanism, but promoting the desorption of $\mathrm{In}$ and $\mathrm{Sb}$ from the $\mathrm{SiO}_{2}$ surface rather than oxygen which is strongly bonded to Si.

For elongated patterns one can notice that their filling depends strongly on the width of the aperture. Whereas $50 \mathrm{~nm}$ and $100 \mathrm{~nm}$ wide apertures are almost completely filled with $\mathrm{InSb}$, scattered islands are observed for larger patterns. These islands are preferably located on the edge of the apertures, this effect being more pronounced for [110] oriented patterns (Fig. 2b). As shown in figure $3 \mathrm{a}$, the island length in [1-10] oriented stripes increases for narrow apertures with a mean value reaching about $5 \mu \mathrm{m}$ for the narrowest ones. In [110] apertures, even in $1 \mu \mathrm{m}$ wide patterns, several micron long islands are observed on the edges of the $\mathrm{SiO}_{2}$ aperture. This phenomenon can be explained considering different diffusion lengths for In adatoms in the two crystallographic orientations and the preferential nucleation of InSb on the edges of the mask apertures. As for the GaSb/GaAs system, the growth of highly mismatched InSb on InAs $(\Delta \mathrm{a} / \mathrm{a}=7.1 \%)$ occurs via a Wolmer Weber growth with the formation of plastically relaxed islands. The density of nucleation sites is governed by the diffusion length of III-element adatoms on the surface of the substrate. In the case of Indium, the adatoms have a larger diffusion length than for Ga. To promote a high density of nucleation sites, one 
should grow InSb with a large growth rate or at low temperature but these conditions are opposite to those needed for selective area MBE where III-element re-evaporation from the dielectric mask is required. In our case, the use of an atomic hydrogen flux allows achieving selectivity at a relatively low growth temperature of $400^{\circ} \mathrm{C}$ with a $0.2 \mathrm{ML} \cdot \mathrm{s}^{-1}$ growth rate. These conditions limit the diffusion length of indium adatoms on the InAs surface but are not sufficient to achieve a $2 \mathrm{D}$ growth mode. However, in sub-micron patterns, the diffusion is further limited by the edges of the mask apertures, increasing the number of nucleation sites, which leads after coalescence to longer InSb islands and a quasi-continuous growth inside sub-100 nm apertures. For large apertures, a smaller diffusion length of In in the [110] direction, particularly near the edge of the aperture, may explain the observed morphologies.

Thanks to their small size, the holes with a $100 \mathrm{~nm}$ diameter are completely filled and InSb islands form nanoscale pyramids delimited by $\{111\}$ facets (Fig. 2d). Interestingly, lateral growth of InSb over the dielectric mask is not homogeneous within the array of holes. As can be seen on figure $2 c$, instead of enlarging each pyramid in the same way, feeding the array with more material leads to the merging of some pyramids resulting in much larger clusters with well-defined facets. This means that Indium diffusion from island to island occurs over the dielectric area separating them to form clusters that minimize the total energy of the system. Growing similar pyramids with the same amount of lateral overgrowth, that could lead to a $2 \mathrm{D}$ InSb layer, would imply an expansion of the high energy $\mathrm{InSb} / \mathrm{SiO}_{2}$ interface as well as larger facet area than those involved in the formation of some high clusters with a limited lateral extension.

Let's turn back to the elongated patterns to study the electrical properties of these InSb nanostructures. As can be seen on figure 3a, several micron long in-plane InSb nanowires can be grown inside sub-100 nm apertures. Measuring their electrical properties independently from the conductivity of the InAs buffer layer is complicated. One possibility is to use the 
selectivity of InAs citric acid based chemical etching to release the InSb NWs after removing the $\mathrm{SiO}_{2}$ mask with dilute $\mathrm{HF}$ (Fig. 3b). The electrical properties can then be measured either by contacting the InSb before full chemical under etching of InAs or by transferring the InSb NW to a host substrate after full under-etching.

Another approach consists in growing the in-plane InSb nanostructures on a semi-insulating $\mathrm{Al}_{0.8} \mathrm{Ga}_{0.2} \mathrm{Sb}$ pseudo-substrate (figure $1 \mathrm{~b}$ ). A cross-section bright field STEM image of an array of in-plane InSb nanostructures of sample $\mathrm{C}$ grown inside $50 \mathrm{~nm} \times 10 \mu \mathrm{m}$ patterns oriented in [110] direction is shown in figure 4. As can be noticed the shape of the InSb nanostructures exhibits well defined facets but that can be quite different from one nanostructure to another. Whereas shape $n^{\circ} 2$ is the most significant for nanostructures that spread almost completely along the aperture, other shapes can be observed for non-completely filled patterns where the amount of material per island is larger. In this latter case, InSb NWs enlarge their volume either by lateral growth over the dielectric mask leading to a triangular shape with (111) facets (shape $n^{\circ} 3$ and $n^{\circ} 4$ ) or by growing out of the plane in one of the (111) directions (shape $n^{\circ} 1$ ). The observation of those different shapes may indicate that their total energy (involving $\mathrm{InSb} / \mathrm{SiO}_{2}$ interfaces and island surface) is quite similar.

The different interfaces of the sample $\mathrm{C}$ are shown in the TEM cross-sections of figure $5 \mathrm{a}, 5 \mathrm{~b}$ and 5c. Between $\mathrm{Al}(\mathrm{Ga}) \mathrm{Sb}$ and $\mathrm{GaAs}$, the mismatch is accommodated with an interface array of $90^{\circ}$ dislocations (dark area in Fig. 5a). Figure 5b displays the thin and continuous pseudomorphic InP layer protecting $\mathrm{AlGaSb}$ from oxidization and from etching with dilute HF during the mask preparation. Figure $5 \mathrm{c}$ is a zoom on the InSb/pseudo-substrate interface of a triangular shaped nanostructure grown inside a $50 \mathrm{~nm}$ wide aperture, and shown in figure 5d. Black spots observed at the interface between InSb and AlGaSb corresponds to the interface dislocations accommodating the $6 \%$ lattice mismatch between InSb and AlGaSb. As can be seen on the chemical EDX analysis of figure 5d, nanostructures are naturally 
composed of In and Sb, and phosphorous has been removed from the aperture surface during the deoxidization process under $\mathrm{Sb}_{2}$ flux.

The electrical properties of an InSb in-plane nanowire can thus be studied without neither any etching of the buffer layer nor any transfer of the nanowire to a host substrate. Figure $6 \mathrm{a}$ and 6b displays plan view SEM and cross-section STEM image of an in-plane [1-10] oriented NW grown inside a $100 \mathrm{~nm} \times 3 \mu \mathrm{m}$ pattern. The rather small length of the pattern leads to a complete filling of the $\mathrm{SiO}_{2}$ aperture. A detail analysis of the electrical properties of such an InSb NW MOSFET with a $3 \mu \mathrm{m}$ gate length oriented in [110] direction is proposed in reference [13]. With the NW presented here (figure 6a and 6b), a MOSFET with a $1 \mu \mathrm{m}$ gate length have been processed (Figs. 6c and 6d) and characterized at 77K (Figs. 6e and 6f). At this temperature, the role of intrinsic charge carriers in the bulk part of the InSb NW is reduced and the conductance is mainly due the accumulation of electrons induced by the electrostatic control of the top gate. A gate voltage larger than $0.5 \mathrm{~V}$ is needed to accumulate electrons in InSb (Fig. 6f). From the $I_{D}=f\left(V_{D S}\right)$ characteristics of figure $6 e$, one can estimate the conductance of the device at $\mathrm{V}_{\mathrm{GS}}=1.5 \mathrm{~V}$ and low drain voltage to be about one quarter of the quantum conductance $\left(2 \times 10^{-5} \mathrm{~S}\right)$. It is however limited by (i) the presence of an oxidized InSb shell around the NW, which limits both the efficiency of the gate electrostatic control and the mobility of electrons in the accumulation regime, and (ii) by the large contact resistances. The former could be alleviated by an appropriate chemical treatment of the InSb surface before ALD [15]. The latter could be reduced using a dummy gate process based on the Hydrogen Silsesquioxane (HSQ) resist and selective area growth of heavily doped InSb access areas as demonstrated recently for InAs NW MOSFET [7].

\section{Conclusion}

In conclusion, the selective area MBE of InSb nanostructures on highly mismatched InAs and AlGaSb pseudo-substrates is demonstrated. We show that the nucleation probability of 
InSb on the pseudo-substrate surface is increased near the edges of the mask apertures, resulting in very long in-plane nanostructures inside sub-100 nm apertures. For very dense patterns, diffusion of Indium can occur over the dielectric mask to promote the formation of high InSb clusters, more inclined to minimize the system energy. Eventually, we show that, with this growth mode applied to a semi-insulating pseudo-substrate, in-plane InSb NW MOSFET with a $1 \mu \mathrm{m}$ gate length can be fabricated without neither any transfer nor any etching of the nanostructure.

\section{Acknowledgments}

The authors would like to acknowledge financial support from the national research agency under the TOPONANO (contract No.: ANR-14-OHRI-0017-03), the French Technological Network Renatech, and the Région Hauts de France.

\section{References}

[1] T.Ashley, L.Buckle, S.Datta, M.T.Emeny, D.G.Hayes, K.P.Hilton, R.Jefferies, T.Martin, T.J.Phillips, D.J.Wallis, P.J.Wilding and R.Chau, Electron. Letters 43 (14), p. (2007)

[2] P. Caroff et al, Small 4, 878 (2008)

[3] H.A. Nilsson et al, Appl. Phys. Lett. 96, 153505 (2010)

[4] S.Gazibegovic et al, Nature 548, 434 (2017)

[5] M.Fahed et al, Nanotechnology 27, 505301 (2016)

[6] M.Fahed et al, J. Crystal Growth 477, 45 (2017)

[7] M.Pastorek et al, 2019 Nanotechnology 30, 035301

[8] M.Friedl at al. 2018 Nano Lett. 18 2666-2671

[9] S.Vaitiekènas, 2018 Phys. Rev. Lett. 121, 147701

[10] V.Chinni et al, 2017 IEEE Journal of the Electron Device Society 5, 53 
[11] Y. Wang, P. Ruterana, L. Desplanque, S. El Kazzi, X. Wallart, Journal of Applied Physics, 109, pp.023509-1-6 (2011)

[12] L.Desplanque et al, Nanotechnology 25 (2014) 465302

[13] L.Desplanque et al, Nanotechnology 29 (2018) 305705

[14] L.Haworth et al, Appl. Surf. Science 166 (2000) 253

[15] C. H. Hou et al, J. Electrochem. Soc. 2008 volume 155, issue 9, G180-G183

\section{Figures captions:}

\section{Figure 1.}

Schemes of the pseudo-substrates used in this study. Sample A and B are grown on InAs pseudo-substrate (a). Sample C is grown on AlGaSb pseudo-substrate (b).

\section{Figure 2.}

Plan view scanning electron micrographs of sample A for different patterns: $50 \mathrm{~nm}$ to $1 \mu \mathrm{m}$ wide and $20 \mu \mathrm{m}$ long [1-10] oriented stripes (a), 50nm to $1 \mu \mathrm{m}$ wide and $20 \mu \mathrm{m}$ long [110] oriented stripes (b) and an array of holes with a $100 \mathrm{~nm}$ diameter (large view (c) and zoom (d)).

\section{Figure 3.}

Evolution of the mean length of the InSb islands with respect to the width of the aperture oriented in [1-10] direction measured from figure 1a (a). Bird view SEM image of a single inplane InSb nanowire of sample B grown inside a 100nmx3 $\mu \mathrm{m}$ aperture and partially released by removing the $\mathrm{SiO}_{2}$ layer with dilute $\mathrm{HF}$ and citric acid based chemical etching of InAs

\section{Figure 4.}


Cross-section bright field STEM image of an array on in-plane InSb NW of sample C. The different shape encountered are numbered from 1 to 4 .

\section{Figure 5.}

TEM characterization of sample C: (a) AlGaSb/AlSb/GaAs interface, (b) $\mathrm{SiO} 2 / \mathrm{InP} / \mathrm{AlGaSb}$ interface, (c) InSb/AlGaSb interface and (d) HAADF and EDX analysis of a triangular nanostructure grown inside a $50 \mathrm{~nm}$ wide aperture.

\section{Figure 5.}

Plan view SEM image of an in-plane InSb nanowire of sample C grown inside a 100nm x $3 \mu \mathrm{m}$ aperture (a) and corresponding cross-section STEM image (b). Bird view SEM image of the NW MOSFET after Ti/Au contact deposition (c) and plan view SEM image after gate oxide and gate electrode deposition $(\mathrm{d}) . \mathrm{I}_{\mathrm{D}}=\mathrm{f}\left(\mathrm{V}_{\mathrm{DS}}\right)(\mathrm{e})$ and transfer characteristics (f) measured on this $1 \mu \mathrm{m}$ gate length InSb NW MOSFET at 77K. 
Figure 1
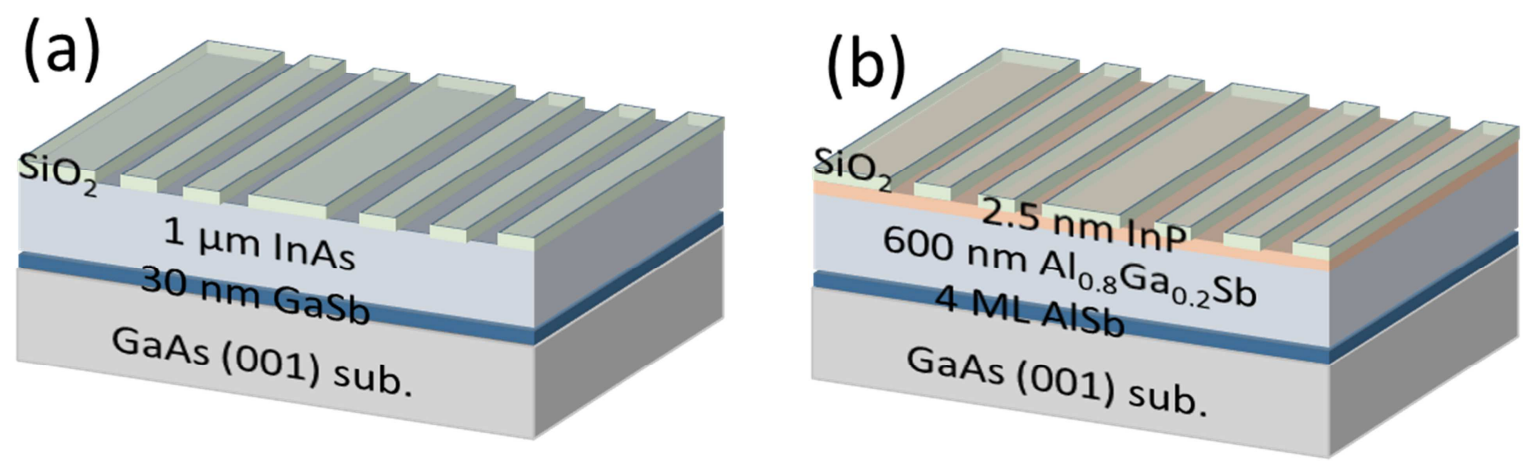
Figure 2

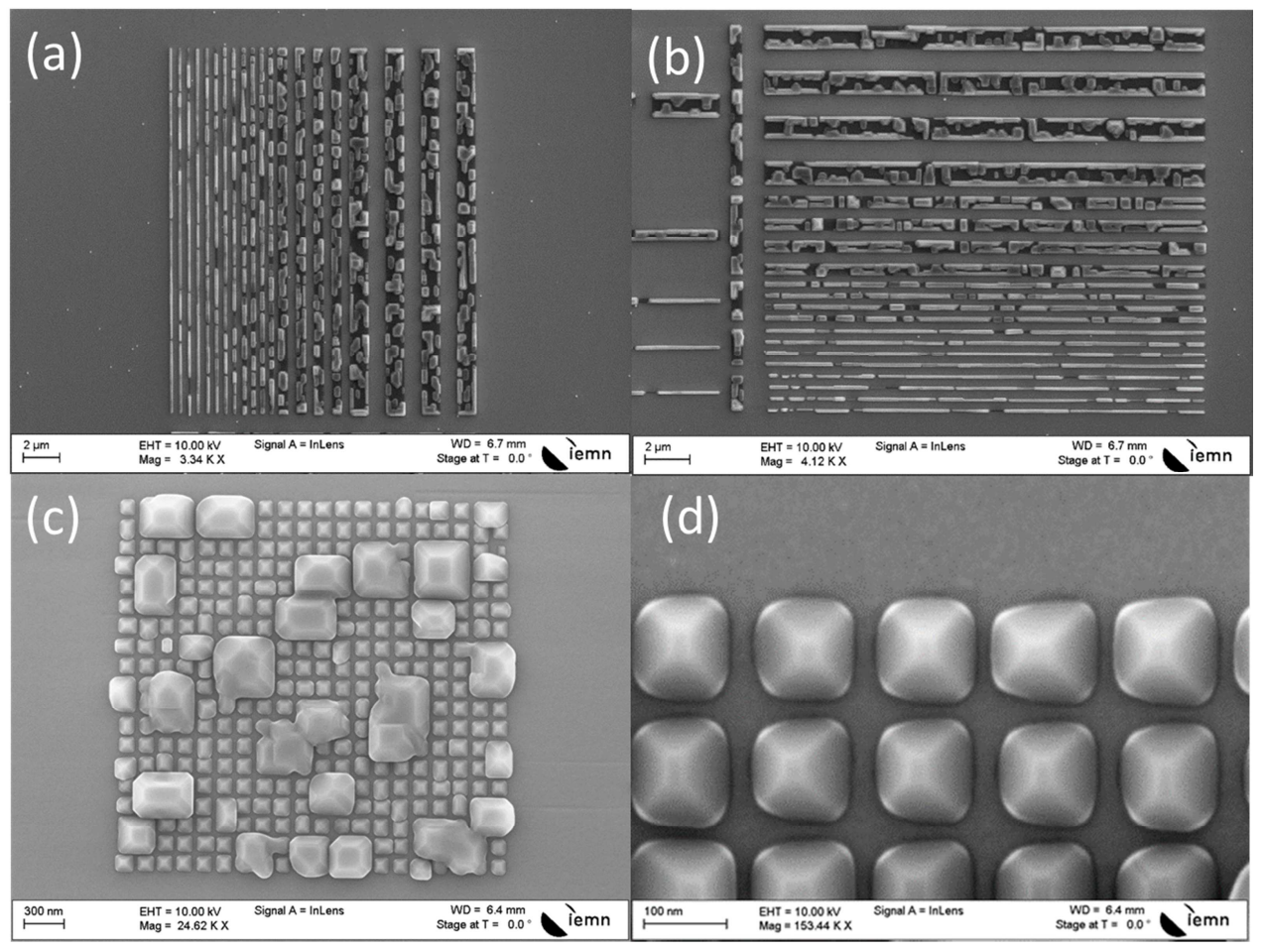


Figure 3
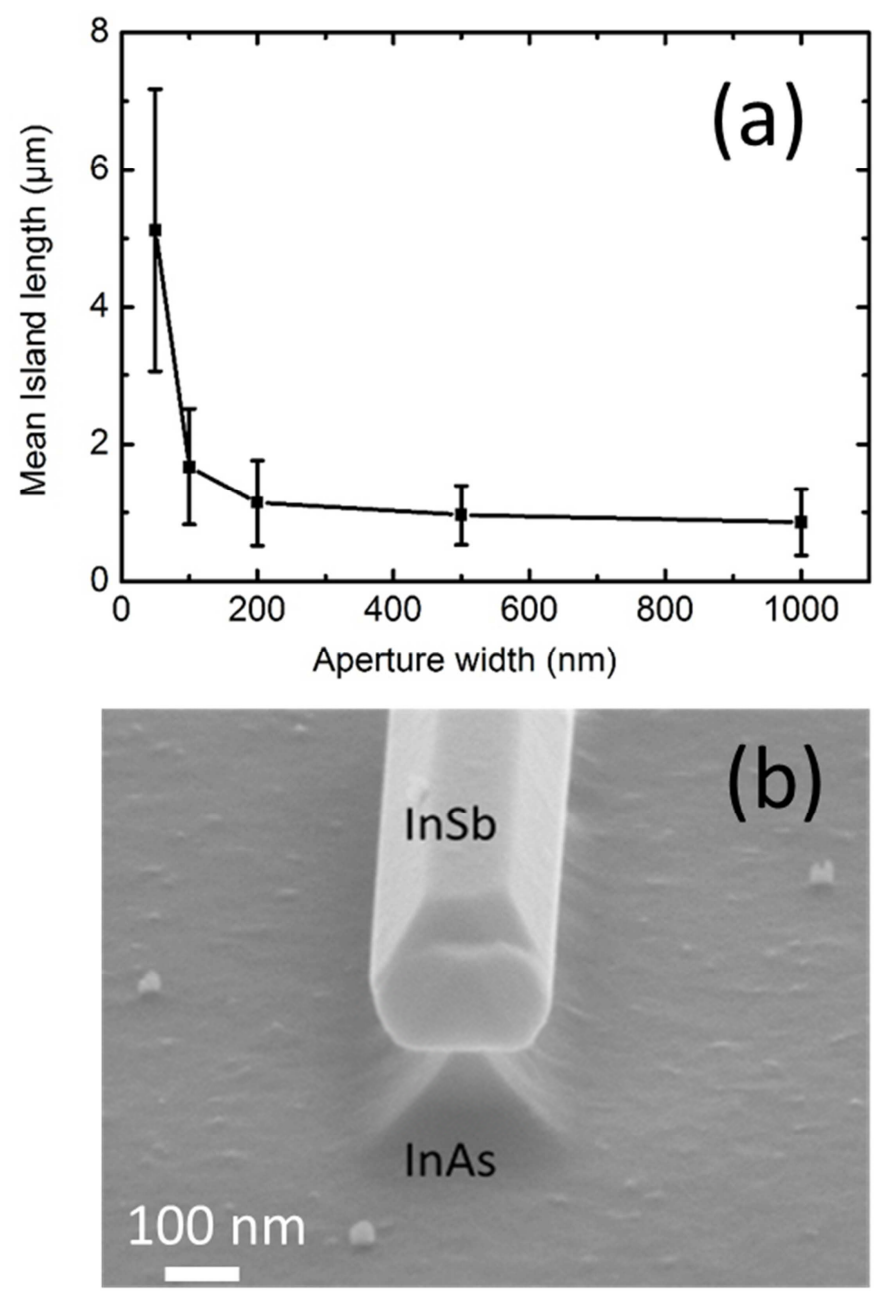
Figure 4

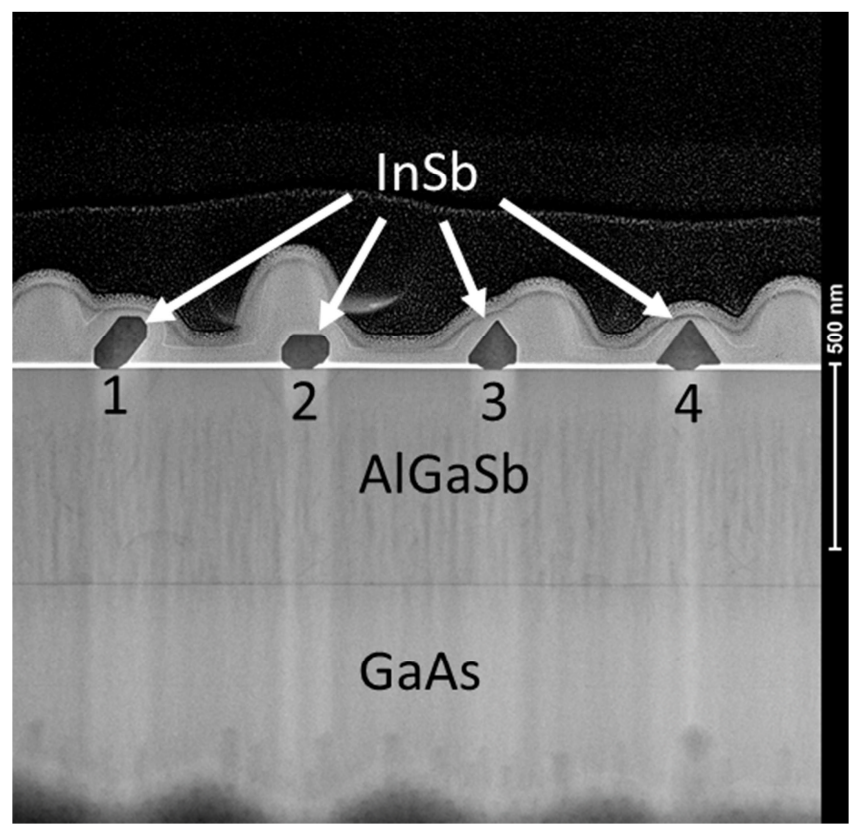

Figure 5

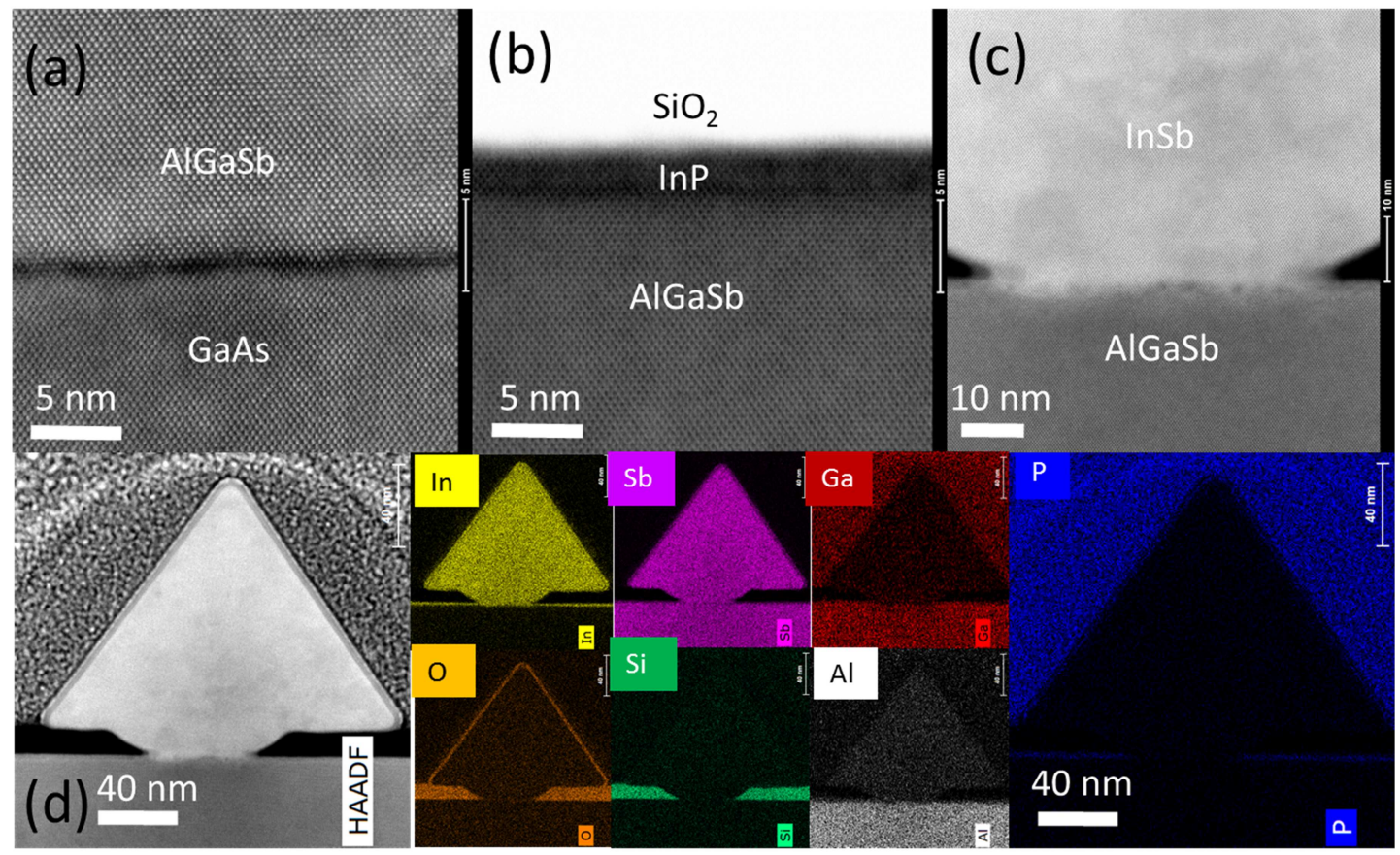


Figure 6
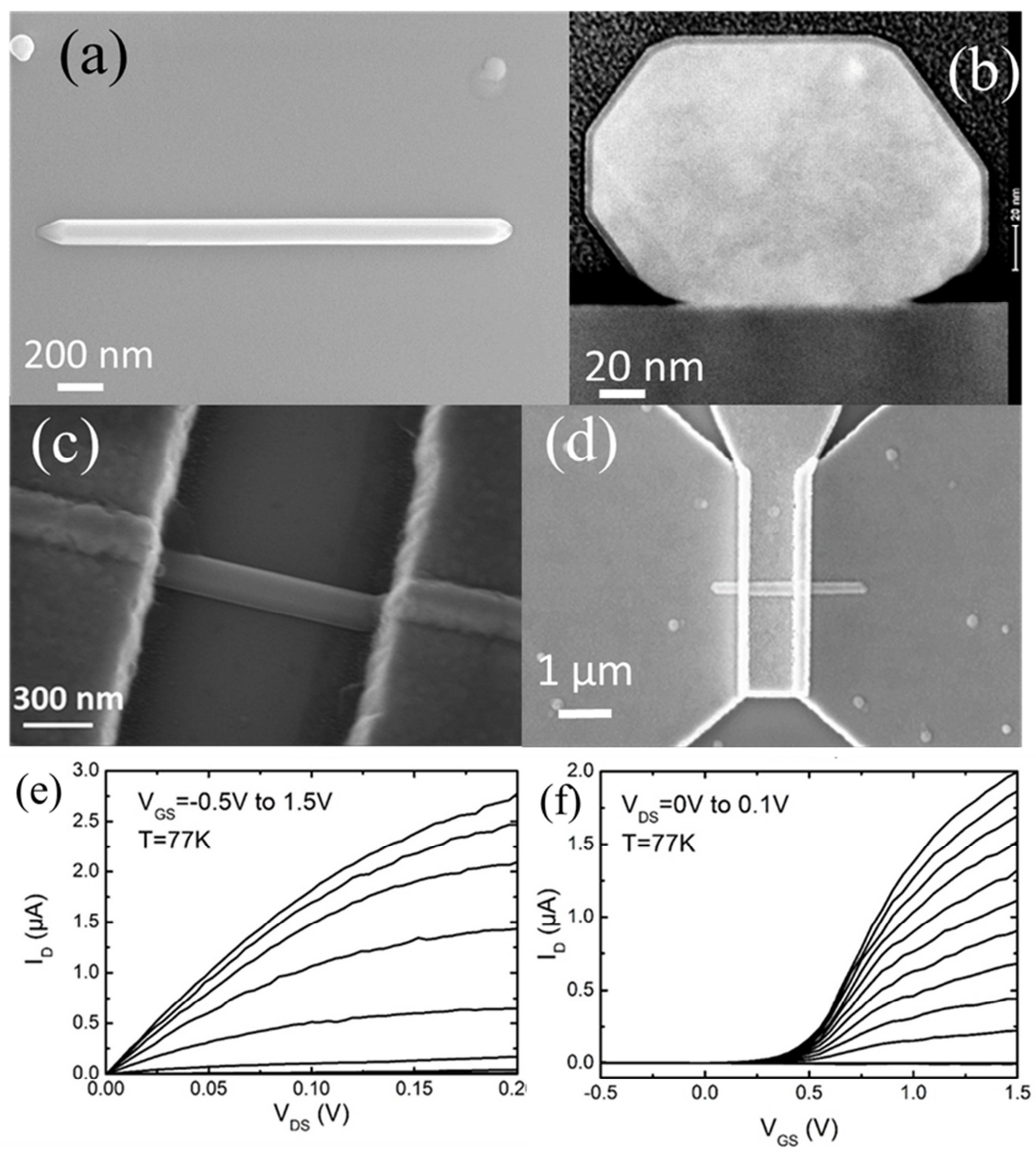